\title{
Perfusão tecidual pode ser utilizada para predição de mortalidade em unidade de terapia intensiva?
}

\author{
Can tissue perfusion be used for predicting mortality in an intensive care unit?
}

\section{Kelser de Souza Kock ${ }^{1}$, Carlos Eduardo Pereira de Carvalho² ${ }^{2}$ Jefferson Luiz Brum Marques ${ }^{3}$}

Kock KS, Carvalho CEP, Marques JLB. Perfusão tecidual pode ser utilizada para predição de mortalidade em unidade de terapia intensiva? / Can tissue perfusion be used for predicting mortality in an intensive care unit? Rev Med (São Paulo). 2020 mar.-abr.;99(2):128-33.

RESUMO: Objetivo: Comparar a acurácia para predição de mortalidade dos indicadores de perfusão tecidual e APACHE II nas primeiras 24 horas de internação em uma unidade de terapia intensiva (UTI) do sul do Brasil. Métodos: coorte prospectiva. Foram avaliados o diagnóstico de internação, idade, sexo, casos cirúrgicos ou não-cirúrgicos, APACHE II, índice de perfusão tecidual (IP), lactato sérico, saturação venosa central de oxigênio $(\mathrm{SvcO} 2)$ e a diferença venosa-arterial de dióxido de carbono $(\triangle \mathrm{PCO} 2)$ nas primeiras 24 horas de internação. Os participantes foram acompanhados até o desfecho na UTI: alta ou óbito. Resultados: Participaram do estudo 126 indivíduos com média de idade de $62,2 \pm 16,8$ anos e principal causa de internação cardiovascular $(37,3 \%)$. As variáveis que estiverem relacionadas ao óbito foram apenas a maior idade $(\mathrm{p}=0,015)$, casos não-cirúrgicos $(\mathrm{p}=0,001)$, maior pontuação no APACHE II $(\mathrm{p}=0,001)$ e maior tempo de internação $(\mathrm{p}=0,004)$. O estudo elaborou um modelo de avaliação prognóstica utilizando os casos não-cirúrgicos e a $\mathrm{SvcO} 2<65 \%$ que, quando comparado com o APACHE II, se mostrou mais acurado para previsão de mortalidade em UTI (área sob a curva ROC 0,727 (IC 95\% $0,616-0,838$ versus 0,802 (IC $95 \% 0,701-0,904)$. Conclusão: Os indicadores de perfusão tecidual analisados não apresentaram relevância estatística como preditores de mortalidade nas primeiras 24 horas quando analisados de forma independente no estudo. O escore APACHE II apresentou-se como uma boa forma de avaliação prognostica para os pacientes, assim como o modelo proposto no presente estudo para previsão de mortalidade, que quando comparado ao escore APACHE II, apresentou um índice de confiabilidade e uma área sob a curva ROC mais elevada.

Descritores: Mortalidade; Cuidados críticos; Unidade de terapia intensiva; APACHE; Ácido láctico; Fotopletismografia; Prognóstico.

\begin{abstract}
Objective: To compare the accuracy of tissue perfusion indicators with APACHE II for predicting mortality in the first 24 hours of hospitalization in an intensive care unit (ICU) in southern Brazil. Methods: A prospective cohort study. The evaluated variables were: hospitalization diagnosis, age, gender, surgical or non-surgical cases, APACHE II, tissue perfusion index (PI), serum lactate, central venous oxygen saturation $(\mathrm{SvcO} 2)$ and arterial-venous carbon dioxide difference $(\triangle \mathrm{PCO} 2)$ in the first 24 hours of hospitalization. Participants were followed-up until the ICU outcomes of discharge or death. Results: A total of 126 subjects with a mean age of $62.2 \pm 16.8$ years having the main cause of cardiovascular hospitalization (37.3\%) participated in the study. The variables which were related to death were only the greater age $(p=0.015)$, non-surgical cases $(p=0.001)$, higher APACHE II score $(\mathrm{p}=0.001)$ and longer hospitalization time $(\mathrm{p}=$ $0.004)$. The study developed a prognostic evaluation model using non-surgical cases and $\mathrm{SvcO} 2<65 \%$, which was more accurate for predicting ICU mortality when compared to APACHE II (area under the ROC curve 0.727 (95\% CI) $0.616-0.838$ versus 0.802 (95\% CI 0.701-0.904). Conclusion: The analyzed tissue perfusion indicators were not statistically significant as predictors of mortality in the first 24 hours when analyzed independently in the study. The APACHE II score showed to be a good form of prognostic evaluation for the patients, as well as the model proposed in the present study for mortality prediction, presenting a higher reliability index and area under the ROC curve when compared to the APACHE II score.
\end{abstract}

Keywords: Mortality; Critical care; Intensive care units; APACHE; Lactic acid; Photoplethysmography; Prognosis.

Universidade do Sul de Santa Catarina (UNISUL), Tubarão, SC, Brasil. Apresentado no XVII Congresso Sul Brasileiro de Medicina Intensiva. Balneário Camboriú, SC, 2017.

1. Professor da Universidade do Sul de Santa Catarina de Tubarão, SC. ORCID: https://orcid.org/0000-0002-0117-6142. Email: kelserkock@yahoo.com.br.

2. Acadêmico no curso de Medicina da Universidade do Sul de Santa Catarina de Tubarão, SC. ORCID: https://orcid.org/0000-0002-3217-7009. Email: cepc@me.com.

3. Professor da Universidade Federal de Santa Catarina (UFSC), SC. ORCID: https://orcid.org/0000-0001-8910-4735. Email: jlbmarques@gmail.com Endereço para correspondência: Kelser de Souza Kock. Rua Galdino José de Bessa, 164 - Bairro Oficinas. Tubarão, SC. CEP: 88702-220. Email: kelserkock@yahoo.com.br 


\section{INTRODUÇÃO}

S centros de terapia intensiva (CTI) destacamenfermos, alta complexidade e elevado custo de serviço. Neste sentido, a utilização de indicadores de prognóstico possibilita uma avaliação do perfil epidemiológico de cada CTI, assim como um rastreamento da probabilidade dos piores desfechos. Dentre os escores mais utilizados, podem ser citados o Acute Physiology and Chronic Health Evaluation II (APACHE II), Sepsis Related Organ Failure Assessment (SOFA), Simplified Acute Physiology Score II (SAPS II) e Logistic Organ Dysfunction System (LODS) ${ }^{1-5}$. Esses escores são calculados através de um somatório de valores numéricos correspondentes a alterações clínicas e laboratoriais apresentadas pelo paciente.

O APACHE II é provavelmente o mais conhecido e usado, sendo introduzido em 1985. Ele consiste na avaliação de doze variáveis clínicas, utilizando-se dos piores valores apresentados nas mesmas nas primeiras 24 horas de internação na UTI ${ }^{1,4,5}$.

A perfusão tecidual também tem sido utilizada como método de avaliação prognóstica e de efetividade de tratamento em pacientes de alto risco ${ }^{6-11}$. A perfusão tecidual pode ser avaliada tanto por sinais clínicos, não invasivos, quanto por critérios laboratoriais e invasivos, que visam a identificação precoce do comprometimento da oxigenação de órgãos e tecidos ${ }^{6-9}$. Os sinais clínicos de hipoperfusão tecidual incluem, entre outros, aumento da frequência cardíaca, tempo de enchimento capilar maior que dois segundos, palidez e diminuição da temperatura de extremidades. Entretanto, os sinais clínicos nem sempre detectam a hipoperfusão com precocidade ou representam de forma precisa o que ocorre na perfusão periférica ${ }^{1,12}$.

Métodos laboratoriais e invasivos também podem ser utilizados na avaliação da perfusão tecidual, sendo os principais a saturação venosa central de oxigênio $\left(\mathrm{SvcO}_{2}\right)$, o nível sérico de lactato, a diferença venosa-arterial de dióxido de carbono $\left(\triangle \mathrm{PCO}_{2}\right)$, o excesso de bases e o índice de perfusão (IP) periférico ${ }^{6-8,11,12}$.

A $\mathrm{SvcO}_{2}$ expressa de modo indireto o consumo de oxigênio pelo corpo, podendo, portanto, ser utilizada como índice de oxigenação tecidual. ${ }^{(1,6,13)} \mathrm{O}$ nível sérico de lactato também é utilizado na avaliação da perfusão tecidual ${ }^{6}$, pois ao acontecer a hipóxia tecidual, a célula, visando manter a sua produção energética, aumenta a produção da glicose anaeróbia, que é uma reação cujo término libera lactato no sangue, aumentando assim o seu nível sérico ${ }^{6,12,14,15}$. $\mathrm{A} \triangle \mathrm{PCO}_{2}$ é calculada através da diferença entre a $\mathrm{PCO}_{2}$ colhido na artéria pulmonar, e a $\mathrm{PCO}_{2}$ arterial, e em condições normais apresenta valores de variam de 2 a 5 $m m \mathrm{mg}^{16,17}$. Por apresentar precocidade em sua alteração, antes de variações na pressão arterial, do nível de lactato sérico e da frequência cardíaca, pode ser utilizada para avaliação da perfusão em pacientes em que haja suspeita de hipoperfusão ${ }^{12}$.

O IP é derivado do sinal fotopletismográfico (PPG) fotoelétrico obtido através da oximetria de pulso, que é uma técnica usada em grande parte das unidades de terapia intensiva, por sua facilidade e custo de realização. A inclusão desse índice no sinal da oximetria de pulso é recente, e seu uso para avaliação precoce da perfusão tecidual, e sua implicação prognóstica ainda necessita de mais estudos ${ }^{8,9}$.

Nesta perspectiva, o objetivo deste trabalho foi comparar a acurácia do APACHE II para predição de mortalidade com os indicadores de perfusão tecidual nas primeiras 24 horas de internação em uma unidade de terapia intensiva do sul de Santa Catarina.

\section{MÉTODOS}

Foi realizada uma coorte prospectiva no centro de terapia intensiva adulto do Hospital Nossa Senhora da Conceição (HNSC), localizado em Tubarão, SC, Brasil.

Foram selecionados indivíduos hospitalizados entre setembro de 2016 e novembro de 2016, cujos familiares assinaram o termo de consentimento livre e esclarecido. Como critérios de exclusão foram considerados os indivíduos transferidos da UTI, má qualidade do sinal de PPG e prontuários incorretos ou com ausência de informações.

Como este é um estudo de acurácia, para o cálculo amostral foi considerada uma prevalência de $25 \%$ de mortalidade $(\mathrm{P}=0,25)$, conforme estimativa prévia, com erro de $10 \%(\mathrm{e}=0,1)$ no intervalo de confiança de $95 \%$ $\left(Z_{\mathrm{a} / 2}=1,96\right)$. A sensibilidade foi definida como $91 \%$ $(\mathrm{Sens}=0,91)$. A equação utilizada está descrita abaixo ${ }^{18}$.

$$
n_{\text {sens }}=\frac{\left(Z_{\alpha / 2}\right)^{2} \text { Sens. }(1-\text { Sens })}{e^{2} \cdot P} .
$$

Para a sensibilidade, $\operatorname{Logo} n_{\text {sens }} \approx 126$.

$\mathrm{Na}$ coleta de dados foram incluídos o diagnóstico de internação, idade, sexo, sinal da curva de pulso de PPG, pontuação na escala APACHE II $^{19} \mathrm{e}$ indicadores de perfusão tecidual: lactato, $\triangle \mathrm{PCO} 2$ e $\mathrm{SvcO} 2$ nas primeiras 24 horas. $\mathrm{O} \triangle \mathrm{PCO} 2$ foi calculado pela diferença da pressão venosa e arterial de oxigênio ( $\mathrm{PvO} 2-\mathrm{PaCO} 2)$. Apenas o sinal de PPG foi coletado a beira do leito. As demais variáveis foram extraídas do prontuário eletrônico. $\mathrm{O}$ indivíduo foi acompanhado até o desfecho: alta ou óbito, contabilizando o tempo de internação na UTI.

A variável IP foi extraída do sinal de PPG por meio de um um oxímetro da marca Reflex Aqwave ${ }^{\mathrm{TM}}$. O mesmo possui capacidade de armazenamento dos valores da curva de pulso numa frequência amostral de $60 \mathrm{~Hz}$ e duração de um minuto. O dispositivo pode exportar os dados em arquivo de texto para análise computacional. Os dados provenientes da curva de pulso de PPG foram manipulados no software MATLAB (Mathworks Inc., USA), para obtenção do índice de perfusão (IP) tecidual. Foi utilizada a equação $\mathrm{IP}=(\mathrm{AC} / \mathrm{DC}) * 100 \%$, onde $\mathrm{AC}$ é o componente pulsátil e DC o componente não pulsátil ${ }^{20,21}$ (Figura 1). 


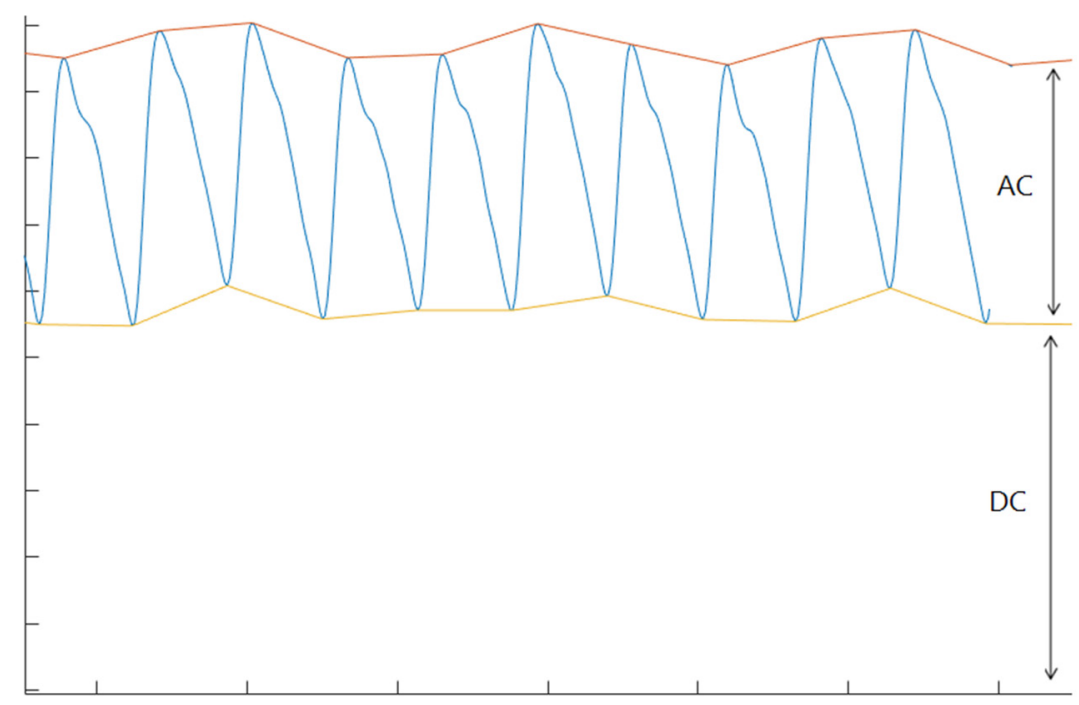

Fonte: Elaboração do autor. AC: Componente pulsátil; DC: Componente não-pulsátil.

Figura 1 - Identificação dos parâmetros AC e DC extraídos do PPG

Este trabalho foi submetido ao Comitê de Ética em Pesquisa da Universidade do Sul de Santa Catarina (UNISUL), Palhoça, SC, Brasil e foi aprovado sob o CAAE 50687515.6.0000.5369.

Os dados foram armazenados em um banco de dados criado com o auxílio do software Excell@, e posteriormente foi exportado para o software SPSS $20.0{ }^{\circledR}$. Os dados foram apresentados por meio de números absolutos e percentuais, medidas de tendência central e dispersão. As variáveis foram comparadas em relação ao desfecho: alta ou óbito por meio de regressão logística bruta e ajustada. Para análise multivariada foram consideradas as variáveis com $\mathrm{p}<0,2$, utilizando o método backward. A variável $\mathrm{SvcO} 2$ foi dicotomizada no ponto de corte $\leq 65 \%$ e $>65 \%$. As demais variáveis de perfusão tecidual foram consideradas contínuas na análise. A acurácia dos modelos multivariados foram comparados com o APACHE II por meio da curva ROC. Foi considerado intervalo de confiança de $95 \%$, com nível de significância estatística de 5\%.

\section{RESULTADOS}

Foram acompanhados 136 indivíduos participantes da pesquisa. Destes, 10 foram excluídos de acordo com os critérios metodológicos. Totalizaram 126 sujeitos, com mediana de idade acima de 60 anos, sendo sexo masculino o mais prevalente. Como diagnósticos mais comuns de internação na UTI, podem ser destacadas as doenças cardiovasculares e neurológicas, com pouco mais da metade dos casos, seguido por neoplasias e infecções. A mediana do tempo de internação foi 6 dias, e aproximadamente um quarto dos participantes acompanhados vieram a óbito (Tabela 1).
Tabela 1 - Características da amostra do CTI da HNSC em Tubarão, SC, Brasil

\begin{tabular}{|c|c|c|}
\hline & Mediana (IQL) & $\overline{n(\%)}$ \\
\hline Idade (anos) & $67,0(51,0-75,0)$ & \\
\hline \multicolumn{3}{|l|}{ Sexo } \\
\hline Masculino & & $71(56,3)$ \\
\hline Feminino & & $55(43,7)$ \\
\hline \multicolumn{3}{|l|}{ Cirúrgico } \\
\hline Não & & $61(48,9)$ \\
\hline $\operatorname{Sim}$ & & $65(51,6)$ \\
\hline \multicolumn{3}{|l|}{ Diagnóstico } \\
\hline Cardiovascular & & $47(37,3)$ \\
\hline Neurológico & & $21(16,7)$ \\
\hline Neoplasia & & $14(11,1)$ \\
\hline Infecção & & $13(10,3)$ \\
\hline Gastrointestinal & & $11(8,7)$ \\
\hline Politrauma & & $11(8,7)$ \\
\hline Respiratório & & $6(4,8)$ \\
\hline Outros & & $3(2,4)$ \\
\hline APACHE II & $13,0(8,0-18,0)$ & \\
\hline $\begin{array}{l}\text { Probabilidade de morte } \\
(\%)\end{array}$ & $16,5(8,7-29,1)$ & , \\
\hline $\begin{array}{l}\text { Tempo de internação } \\
\text { (dias) }\end{array}$ & $6,0(3,0-15,0)$ & \\
\hline \multicolumn{3}{|l|}{ Óbito } \\
\hline Não & & $95(75,4)$ \\
\hline Sim & & $31(24,6)$ \\
\hline
\end{tabular}

As variáveis relacionadas à perfusão tecidual nas primeiras 24 horas de internação na UTI estão demonstradas na Tabela 2.

As variáveis que estiverem relacionadas ao óbito foram a maior idade, casos não-cirúrgicos, maior pontuação no APACHE II e maior tempo de internação (Tabela 3). 
Kock KS, et al. Perfusão tecidual pode ser utilizada para predição de mortalidade em UTI?

Tabela 2 - IP, Lactato, $\mathrm{SvcO} 2$ e $\triangle \mathrm{PCO} 2$ nas primeiras 24 horas de internação

\begin{tabular}{lcc}
\hline & Mínimo - Máximo & Mediana (IQL) \\
\hline IP $(\%)$ & $47,3-231,9$ & $102,9(83,2-134,6)$ \\
Lactato (mmol/L) & $4,2-46,7$ & $14,0(8,2-21,8)$ \\
SvcO2 (\%) & $30,0-95,0$ & $67,0(62,0-78,0)$ \\
$\Delta \mathrm{PCO} 2(\mathrm{mmHg})$ & $0,0-22,0$ & $9,0(6,0-11,0)$ \\
\hline
\end{tabular}

Legenda: IQL: Distância interquartílica

Tabela 3 - Análise bivariada e odds ratio bruto para o óbito

\begin{tabular}{|c|c|c|c|c|}
\hline & $\begin{array}{c}\text { ALTA } \\
\text { Mediana (IQL) }\end{array}$ & $\begin{array}{c}\text { ÓBITO } \\
\text { Mediana (IQL) }\end{array}$ & OR $(\mathrm{IC}-95 \%)$ & $\mathbf{p}$ \\
\hline Idade (anos) & $65(48-72)$ & $74(57-78)$ & $1,038(1,007-1,069)$ & 0,015 \\
\hline $\begin{array}{l}\text { Sexo* } \\
\text { Feminino } \\
\text { Masculino }\end{array}$ & $\begin{array}{l}55(77,5) \\
39(72,2)\end{array}$ & $\begin{array}{l}16(22,5) \\
15(27,8)\end{array}$ & $\begin{array}{c}1,000 \\
0,756(0,335-1,709)\end{array}$ & 0,502 \\
\hline $\begin{array}{l}\text { Cirúrgico* } \\
\text { Sim } \\
\text { Não }\end{array}$ & $\begin{array}{l}57(89,1) \\
37(60,7)\end{array}$ & $\begin{array}{c}7(10,9) \\
24(39,3)\end{array}$ & $\begin{array}{c}1,000 \\
5,282(2,067-13,496)\end{array}$ & 0,001 \\
\hline APACHE II & $11(7-17)$ & $17(12-23)$ & $1,130(1,055-1,211)$ & 0,001 \\
\hline Tempo de internação (dias) & $4(3-11)$ & $15(6-27)$ & $1,038(1,012-1,064)$ & 0,004 \\
\hline IP & $109(84-140)$ & $109(84-135)$ & $0,737(0,236-2,304)$ & 0,599 \\
\hline Lactato $(\mathrm{mmol} / \mathrm{L})$ & $14(8-22)$ & $16(8-21)$ & $0,984(0,942-1,028)$ & 0,470 \\
\hline $\mathrm{SvcO} 2(\%)$ & $68(63-77)$ & $65(58-79)$ & $0,986(0,946-1,028)$ & 0,500 \\
\hline $\begin{array}{l}\leq 65 \% \\
>65 \%\end{array}$ & $\begin{array}{l}30(61,2) \\
60(77,9)\end{array}$ & $\begin{array}{l}19(38,8) \\
17(22,1)\end{array}$ & $\begin{array}{c}1,00 \\
0,458(0,171-1,229)\end{array}$ & 0,121 \\
\hline$\triangle \mathrm{PCO} 2(\mathrm{mmHg})$ & $8(6-11)$ & $9(6-12)$ & $0,989(0,877-1,117)$ & 0,863 \\
\hline
\end{tabular}

Legenda: * variáveis categóricas expressas em n(\%); IQL: distância interquartílica

Na análise multivariada para predição de mortalidade em UTI, foram propostos dois modelos onde as variáveis apresentaram associação estatística. Comparando o modelo 1 com o APACHE II, pode-se verificar que o mesmo é mais acurado para previsão de mortalidade em UTI, demonstrando área sob a curva ROC de 0,802 (IC

\begin{tabular}{ccc}
\hline Modelo 1 & $\begin{array}{c}\text { OR } \\
(\text { IC }-\mathbf{9 5 \%})\end{array}$ & $\mathbf{p}$ \\
\hline Não-Cirúrgico & $\begin{array}{c}11,895 \\
(3,332-42,473) \\
\text { SvcO2 } \leq 65 \%\end{array}$ & $<0,001$ \\
& $\begin{array}{c}4,229 \\
(1,197-14,935)\end{array}$ & 0,025 \\
\hline Modelo 2 & OR & $\mathbf{p}$ \\
\hline Não-Cirúrgico & $(\mathbf{I C}-\mathbf{9 5 \% )}$ & 0,002 \\
SvcO2 $\leq 65 \%$ & $(2,278-32,938)$ & 0,019 \\
APACHE II & 4,983 & 0,034 \\
\hline
\end{tabular}

Legenda: OR: Odds Ratio

$95 \% 0,701-0,904)$ com $\mathrm{p}<0,001$, enquanto o APACHE II apresentou área soba a curva ROC de 0,727 (IC 95\% $0,616-0,838$ ) com $p=0,002$. Associando o APACHE II ao modelo 1, foi proposto o modelo 2 que, apresentou uma maior acurácia, com área sob a curva de ROC 0,830 (IC $95 \%$ 0,741 - 0,918) com $\mathrm{p}<0,001$ (Figura 2)

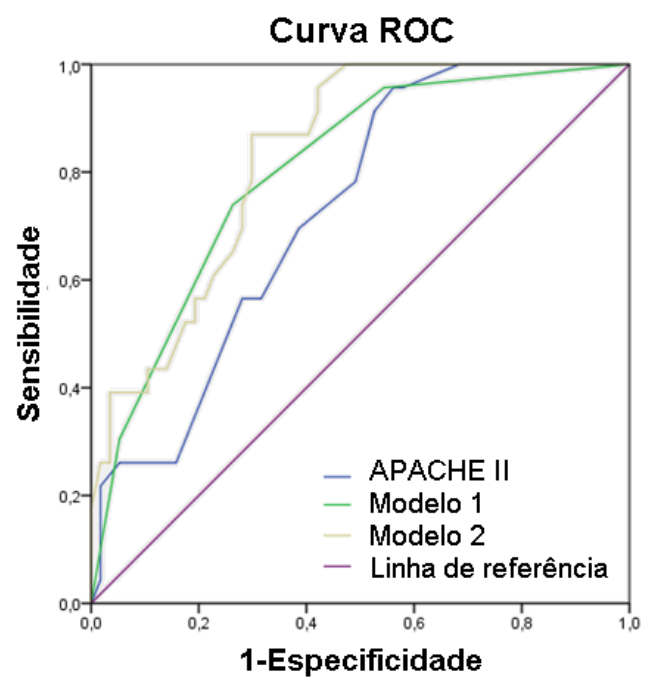

Figura 2 - Modelos de predição de mortalidade baseado em variáveis epidemiológicas e perfusão periférica e Curva ROC: APACHE II $x$ modelo 1 e modelo 2 


\section{DISCUSSÃO}

No presente estudo, a média de idade encontrada representa um perfil de pacientes idosos, sendo que ocorreu uma diferença significativa no grupo de pacientes que evoluíram para óbito ou alta durante a internação, dados que vão de acordo com o estudo realizado por Ho et al. ${ }^{22}$. Porém, o estudo de Williams et al. ${ }^{23}$ realizado com 22298 pacientes não associou a idade ao risco de óbito, indicando que é possível que a idade possa estar associada a outros fatores que contribuem para a evolução negativa do paciente, como maior gravidade da doença e pior estado funcional anterior à internação.

$\mathrm{O}$ número de pacientes do sexo masculino foi superior quando comparado com os indivíduos do sexo feminino, resultado semelhante ao obtido por Nogueira et al. ${ }^{24}$ e Ho et al. ${ }^{22}$. Com relação ao tempo de internação médio obtido não há um consenso em relação ao tempo de internação mínimo para ser considerada uma internação prolongada, variando entre $3 \operatorname{dias}^{23}, 10 \operatorname{dias}^{23}$ e $14 \operatorname{dias}^{25,26}$. Essa variação entre os estudos poderia ser explicada devido ao fato da maioria dos mesmos terem sidos realizados em unidades de terapia intensiva com população clínica e cirúrgica, como é o caso do presente estudo. Foi obtida uma diferença significativa no que se refere ao tempo de internação dos pacientes com evolução para óbito $(19,6)$ em relação aos casos que evoluíram para alta $(8,8)$, assim como ocorreu nos estudos realizados por Nogueira et al. ${ }^{24}$ e Santana et al..$^{25}$.

O diagnóstico de internação mais comum entre os pacientes estudados foram as doenças cardiovasculares, sendo aproximadamente metade dos cirúrgicos, resultados semelhantes aos obtidos no estudo de Ho et al. ${ }^{22}$, onde as doenças cardiovasculares foram responsáveis por 33,2\% dos pacientes internados na unidade estudada, e no estudo de Nogueira et al. ${ }^{24}$, onde dos 148 casos analisados, 56 foram devido a doenças do aparelho circulatório.

O APACHE II médio obtido no presente estudo foi similar ao estudo de Oliveira et al. ${ }^{27}$, onde foram analisados 401 pacientes admitidos em uma unidade de terapia intensiva e o APACHE II médio apresentado foi de 12, demonstrando uma diferença estatisticamente significante entre os pacientes com evolução para óbito ou alta. Esses resultados corroboram a efetividade do uso de escore APACHE II como preditor de mortalidade nas unidades de terapia intensiva, como também acontece em diversos estudos $^{1,2,4,5,22,23}$.

Os casos não cirúrgicos apresentaram um risco de mortalidade maior quando comparado aos casos cirúrgicos, sendo um resultado esperado e identificado no trabalho de Hissa et al. ${ }^{2}$.

No presente estudo, os marcadores de perfusão tecidual, sendo eles, IP, $\triangle \mathrm{PCO} 2, \mathrm{SvcO} 2$ e lactato sérico, não apresentaram diferença significativa entre os pacientes com evolução para alta ou óbito. Esse resultado difere do apresentado pelo estudo de Haijar et al. ${ }^{6}$, onde foram analisados o lactato e o excesso de bases na admissão de 1129 pacientes com sepse severa, choque séptico ou pósoperatório de cirurgia de alto risco em uma unidade de terapia intensiva, e foi demonstrado que essas variáveis poderiam ser usadas como preditores de óbitos de forma independente. Outros estudos, também corroboram o uso do lactato e do excesso de bases como preditores de um pior prognóstico em pacientes internados em um centro de terapia intensiva ${ }^{11,28,29}$. Talvez, pela heterogeneidade da amostra, os marcadores de perfusão tecidual não tenham demonstrado diferença significativa entre os grupos alta e óbito.

O IP, uma das variáveis analisadas no estudo, apresenta como vantagem o fato de ser uma forma de avaliação de perfusão tecidual, conforme demonstrado pelos estudos de $\mathrm{He}^{9}$ e Lima et al. ${ }^{9}$, que pode ser obtida de maneira não invasiva, ao contrário da $\triangle \mathrm{PCO} 2$, do $\mathrm{SvcO} 2$ e do lactato sérico, necessitando apenas a utilização de dados obtidos através de um oxímetro de pulso, ainda que, no presente estudo, não tenha apresentado uma diferença estatística significativa entre os grupos estudados.

Utilizando-se de uma variável epidemiológica, caso cirúrgico ou não, junto com a $\mathrm{SvcO} 2$, o presente estudo elaborou um modelo de previsão de mortalidade em unidades de terapia intensiva, que se mostrou mais acurado quando comparado aos resultados encontrados com utilização do escore APACHE II. Este resultado demonstra que outras ferramentas preditivas podem ser utilizadas em UTI com boa precisão.

Como limitação do estudo, pode-se descrever o possível viés na coleta de informações em prontuários e a heterogeneidade da amostra.

\section{CONCLUSÃO}

O presente estudo demostrou que o escore APACHE II apresenta-se como uma boa forma de avaliação prognostica para os pacientes de uma unidade de terapia intensiva, assim como o modelo proposto para previsão de mortalidade utilizando variáveis epidemiológicas (caso cirúrgico ou não cirúrgico) associado a $\mathrm{SvcO} 2$, que quando comparado ao escore APACHE II, apresentou um índice de confiabilidade e uma área sob a curva ROC mais elevada.

Os indicadores de perfusão tecidual analisados neste estudo, sendo eles IP, $\triangle \mathrm{PCO} 2, \mathrm{SvcO} 2$ e o Lactato, não apresentaram relevância estatística como preditores de mortalidade nas primeiras 24 horas quando analisados de forma independente no estudo. 
Kock KS, et al. Perfusão tecidual pode ser utilizada para predição de mortalidade em UTI?

Participação dos autores: Kock $K S$ : proposta da temática do estudo, análise estatística, descrição dos resultados, revisão do artigo. Carvalho CEP: redação do artigo, coleta de dados. Marques JLB: análise estatística, correção e revisão final do artigo.

\section{REFERENCIAS}

1. Vincent JL, Moreno R. Clinical review: scoring systems in the critically ill. Crit Care. 2010;14(2):207. doi: 10.1186/cc8204.

2. Hissa PNG,Hissa MRN,Araújo PSR. Análise comparativa entre dois escores na previsão de mortalidade em unidade terapia intensiva. Rev Bras Clin Med. 2013;11(1):21-6. Disponível em: http://files.bvs.br/ upload/S/1679-1010/2013/v11n1/a3383.pdf.

3. Dehghani A, Abdeyazdan G, Davaridolatabadi E. An overview of the predictor standard tools for patient weaning from mechanical ventilation. Electron Phys. 2016;8(2):1955-63. doi: 10.19082/1955.

4. Chiavone PA, Sens YAS. Evaluation of APACHE II system among intensive care patients at a teaching hospital. Sao Paulo Med J. 2003;121(2):53-7. doi: 10.1590/s1516-31802003000200004.

5. Naqvi IH, Mahmood K, Ziaullaha S, Kashif SM, Sharif A. Better prognostic marker in ICU -APACHE II, SOFA or SAP II! Pak J Med Sci. 2016;32(5):1146-51. doi: 10.12669/pjms.325.10080.

6. Hajjar LA, Nakamura RE, Almeida JP, Fukushima JT, Hoff PMG, Vincent JL, et al. Lactate and base deficit are predictors of mortality in critically ill patients with cancer. Clinics. 2011;66(12):2037-42. doi.org/10.1186/cc10166

7. Westphal GA, Gonçalves AR, Caldeira Filho M, Silva E, Salomão $\mathrm{R}$, Bernardo WM, et al. Guidelines for treatment of severe sepsis/ septic shock: tissue perfusion assessment. Rev Bras Ter Intensiva. 2011;23(1):6-12.

8. He H, Long Y, Liu D, Wang X, Zhou X. Clinical classification of tissue perfusion based on the central venous oxygen saturation and the peripheral perfusion index. Crit Care. 2015;19:330. doi:10.1186/ s13054-015-1057-8

9. Lima AP, Beelen P, Bakker J. Use of a peripheral perfusion index derived from the pulse oximetry signal as a noninvasive indicator of perfusion. Crit Care Med. 2002;30(6):1210-3 doi:10.1097/00003246200206000-00006

10. Lima A, Bakker J. Noninvasive monitoring of peripheral perfusion. Intensive Care Med. 2005;31(10):1316-26. doi: 10.1007/s00134005-2790-2

11. Santos MC, Boniatti MM, Lincho CS, Pellegrini JAS, Vidart J, Rodrigues Filho EM, et al. Inflammatory and perfusion markers as risk factors and predictors of critically ill patient readmission. Rev Bras Ter Intensiva. 2014 Apr-Jun;26(2):130-6. doi: 10.5935/0103507x.20140019

12. Figueiredo LFP, Silva E, Corrêa TD. Avaliação hemodinâmica macro e micro-circulatória no choque séptico. Rev Med. 2008;87(2):84-91.

13. Antonelli M,Levy M,Andrews PJ, Chastre J,HudsonLD, Manthous $\mathrm{C}$, et al. Hemodynamic monitoring in shock and implications for management. In: International Consensus Conference, Paris, France, 27-28April 2006. Intens Care Med. 2007;33(4):575-90. doi: 10.1007/ s00134-007-0531-4.

14. De BackerD. Lactic acidosis. Intens Care Med. 2003;29(5):699-702. doi: 10.1007/s00134-003-1746-7.

15. Valenza F, Aletti G, Fossali T, Chevallard G, Sacconi F, Irace M, et al. Lactate as a marker of energy failure in critically ill patients: hypothesis. Crit Care. 2005;9(6):588-93. doi: 10.1186/cc3818.

16. Vallet B, Teboul JL, Cain S, Curtis S. Venoarterial CO(2) difference during regional ischemic or hypoxic hypoxia. J Appl Physiol. 2000;89(4):1317-21. doi: 10.1152/jappl.2000.89.4.1317.
17. Lamia B, Monnet X, Teboul JL. Meaning of arteriovenous PCO2 difference in circulatory shock. Minerva Anestesiol. 2006;72(6):597604.Availble from: https://www.minervamedica.it/en/getfreepdf/Wf QhrGhmnljBHHkiE\%252FRoUWzRtXEixasXvEyEfzCKfWgZov 9FQkuuSOPWnEDlyluYGgszmUn8zmk\%252BaDDwVv1N4Q\% 253D\%253D/R02Y2006N06A0597.pdf.

18. Hajian-Tilaki K. Sample size estimation in diagnostic test studies of biomedical informatics. J Biomed Inform. 2014;48:193-204. doi: 10.1016/j.jbi.2014.02.013.

19. Knaus WA, Draper EA, Wagner DP, Zimmerman JE. APACHE II: a severity of disease classification system. Crit Care Med. 1985;13(10):818-29.

20. Sandroni C, Cavallaro F, Marano C, Falcone C, De Santis P, Antonelli M. Accuracy of plethysmographic indices as predictors of fluid responsiveness in mechanically ventilated adults: a systematic review and meta-analysis. Intens Care Med. 2012;38(9):1429-37. doi: 10.1007/s00134-012-2621-1

21. Tamura T, Maeda Y, Sekine M, Yoshida M. Wearable photoplethysmographic sensors - past and present. Electronics. 2014;3(2):282-302. doi :10.3390/electronics3020282.

22. Ho KM, Lee KY, Williams T, Finn J, Knuiman M, Webb SAR. Comparison of Acute Physiology and Chronic Health Evaluation (APACHE) II score with organ failure scores to predict hospital mortality. Anaesthesia. 2007;62(5):466-73. doi: 10.1111/j.13652044.2007.04999.x.

23. Williams TA, Ho KM, Dobb GJ, Finn JC, Knuiman M, Webb SAR. Effect of length of stay in intensive care unit on hospital and long-term mortality of critically ill adult patients. Br JAnaesth. 2010;104(4):45964. doi: $10.1093 / \mathrm{bja} / \mathrm{aeq} 025$.

24. Nogueira LS, Santos MR, Mataloun SE, MoockM. Nursing Activities Score: comparação com o Índice APACHE II e a mortalidade em pacientes admitidos em unidade de terapia intensiva. Rev Bras Ter Intensiva. 2007;19(3):327-30. https://doi.org/10.1590/S0103507X2007000300010.

25. Santana-Cabrera L, Torrent RL, Sánchez-Palacios M, RodríguezEscot C, Martín-Santana JD, Hernández Hernández JR. Long-term outcome factors associated with prolonged admission to the ICU. Int J Crit Illn Inj Sci. 2014;4(4):320-1. doi: 10.4103/2229-5151.147558.

26. Laupland KB, Kirkpatrick AW, Kortbeek JB, Zuege DJ. Long-term mortality outcome associated with prolonged admission to the ICU. Chest. 2006;129(4):954-9. doi: 10.1378/chest.129.4.954.

27. Oliveira ABF, Dias OM, Mello MM, Araújo S, Dragosavac D, Nucci A, et al. Factors associated with increased mortality and prolonged length of stay in an adult intensive care unit. Rev Bras Ter Intensiva. 2010;22(3):250-6. https://doi.org/10.1590/S0103$507 X 2010000300006$

28. Kruse O, Grunnet N, Barfod C. Blood lactate as a predictor for inhospital mortality in patients admitted acutely to hospital: a systematic review. Scand J Trauma Resusc Emerg Med. 2011;19:74.. doi: 10.1186/1757-7241-19-74.

29. Husain FA, Martin MJ, Mullenix PS, Steele SR, Elliott DC. Serum lactate and base deficit as predictors of mortality and morbidity. Am J Surg. 2003;185(5):485-91. doi: 10.1016/ s0002-9610(03)00044-8.

Recebido: 02.07.2018

Aceito: 23.03.2020 